TI 2011-089/1

Tinbergen Institute Discussion Paper

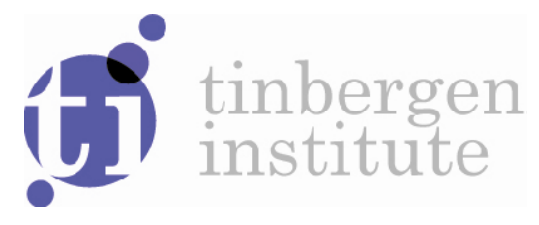

\title{
An Owen-Type Value for Games with Two-Level Communication Structures
}

René van den Brinka

Anna Khmelnitskayab

Gerard van der Laana

a VU University Amsterdam, and Tinbergen Institute;

${ }^{b}$ Faculty of Applied Mathematics, Saint-Petersburg State University. 
Tinbergen Institute is the graduate school and research institute in economics of Erasmus University Rotterdam, the University of Amsterdam and VU University Amsterdam.

More TI discussion papers can be downloaded at http://www.tinbergen.nl

Tinbergen Institute has two locations:

Tinbergen Institute Amsterdam

Gustav Mahlerplein 117

1082 MS Amsterdam

The Netherlands

Tel.: +31(0)205251600

Tinbergen Institute Rotterdam

Burg. Oudlaan 50

3062 PA Rotterdam

The Netherlands

Tel.: +31(0)10 4088900

Fax: +31(0)104089031

Duisenberg school of finance is a collaboration of the Dutch financial sector and universities, with the ambition to support innovative research and offer top quality academic education in core areas of finance.

DSF research papers can be downloaded at: http://www.dsf.nl/

Duisenberg school of finance

Gustav Mahlerplein 117

1082 MS Amsterdam

The Netherlands

Tel.: +31(0)20 5258579 


\title{
An Owen-type value for games with two-level communication structures ${ }^{1}$
}

\author{
René van den Brink ${ }^{2}$ Anna Khmelnitskaya ${ }^{3}$ Gerard van der Laan ${ }^{4}$
}

June 21, 2011

\footnotetext{
${ }^{1}$ This research was supported by NWO (The Netherlands Organization for Scientific Research) grant NL-RF 047.017.017. The research of Anna Khmelnitskaya was also partially supported by CRT Foundation and the University of Eastern Piedmont at Alessandria through the Lagrange Project 2010.

${ }^{2}$ J.R. van den Brink, Department of Econometrics and Tinbergen Institute, VU University, De Boelelaan 1105, 1081 HV Amsterdam, The Netherlands. E-mail: jrbrink@feweb.vu.nl

${ }^{3}$ A.B. Khmelnitskaya, Saint-Petersburg State University, Faculty of Applied Mathematics, Universitetskii prospekt 35, 198504, Petergof, Saint-Petersburg, Russia. Email: a.khmelnitskaya@math.utwente.nl

${ }^{4}$ G. van der Laan, Department of Econometrics and Tinbergen Institute, VU University, De Boelelaan 1105, 1081 HV Amsterdam, The Netherlands. E-mail: glaan@feweb.vu.nl
} 


\begin{abstract}
We introduce an Owen-type value for games with two-level communication structures, being structures where the players are partitioned into a coalition structure such that there exists restricted communication between as well as within the a priori unions of the coalition structure. Both types of communication restrictions are modeled by an undirected communication graph, so there is a communication graph between the unions of the coalition structure as well as a communication graph on the players in every union. We also show that, for particular two-level communication structures, the Owen value and the Aumann-Drèze value for games with coalition structures, the Myerson value for communication graph games and the equal surplus division solution appear as special cases of this new value.
\end{abstract}

Keywords: TU game, coalition structure, communication graph, Owen value, Myerson value.

JEL code: C71 


\section{Introduction}

The study of TU games with coalition structures was initiated in the 1970's, first by Aumann and Drèze [1] and then Owen [10]. In these papers a coalition structure is given by a partition of the set of players. Later this approach was extended in Winter [13] to games with level structures. Another model of a game with limited cooperation presented by means of communication graphs was introduced in Myerson [9]. Various studies in both directions were done during the last three decades, but mostly either within one model or another. Vázquez-Brage, García-Jurado and Carreras [12] is the first study that combines both models by considering a TU game endowed with independent of each other both a coalition structure and a communication graph on the set of players. For this class of games they propose a solution by applying the Owen value for games with coalition structures to the Myerson restricted game of the game with communication graph.

Recently, Khmelnitskaya [7] and Kongo [8] independently from each other have introduced another model of a TU game endowed with both a coalition structure and communication graph, the so-called games with two-level communication structures. In contrast to [12], in this model a two-level communication structure relates fundamentally to the given coalition structure and consists of a communication graph on the collection of the a priori unions in the coalition structure, as well as a communication graph within every union. It is assumed that communication is only possible either among the a priori unions or among single players within an a priori union. No communication and therefore no cooperation is allowed between single players from distinct elements of the coalition structure. Different from the framework of Khmelnitskaya, Kongo reduces the model to a one-level communication model using a special assumption concerning the ability of players to cooperate under the two-level communication structure, namely a set of players is able to cooperate either if it is a connected component within an a priori union or the set of players is the union of at least two connected a priori unions, independently whether the players are connected inside the a priori union they belong to or not.

In this paper we abide by the Khmelnitskaya's framework but we weaken the assumption concerning communication on the upper level between a priori unions allowing for one a priori union among connected unions to be represented by a proper subcoalition. We introduce a new solution for the class of games with two-level communication structures. Different from the solution concepts given in [7] and [8], the new solution is an Owen-type value in the sense that it modifies the Owen value for games with two-level communication structures. As in Owen [10], the payoffs of the players are determined by applying the Shapley value twice. First, the Shapley value is applied to the Myerson restricted game (with respect to the communication graph between unions) of Owen's quotient game between the unions. This gives for each union the total payoff to the players 
of the union. To obtain the individual payoffs, within each union the Shapley value is applied to a game on the players within the union. To construct the game within a union, first a game is obtained by applying Owen's procedure to find such a game but taking account of the communication graph between the unions. Next we construct a restriction of this game taking into account the communication graph within the union and apply the Shapley value to this restriction.

The new Owen-type value for the class of games with two-level communication structures is characterized by four axioms, two on the level of the communication graph between the a priori unions, and two on the level of the communication graphs within the a priori unions. We also show that the Owen value and the Aumann-Drèze value for games with coalition structures, the Myerson value for communication graph games and the equal surplus division solution appear as special cases of this new value for particular two-level communication structures.

The paper is organized as follows. Basic definitions and notation are introduced in Section 2. Section 3 is devoted to the axioms that we require from a solution for games with two-level communication structures. In Section 4 we define an Owen-type value for such games and show that it is the unique solution satisfying these axioms. In Section 5 we consider several special cases and show that the new solution generalizes some well-known solutions for games in coalition structure and communication graph games.

\section{Preliminaries}

\section{$2.1 \quad$ TU games and values}

A situation in which a finite set of players can obtain certain payoffs by cooperating can be described by a cooperative game with transferable utility, or simply a TU game, being a pair $\langle N, v\rangle$, where $N \subset \mathbb{N}$ is a finite set of $n \geq 2$ players and $v: 2^{N} \rightarrow \mathbb{R}$ is a characteristic function on $N$ such that $v(\emptyset)=0$. For any coalition $S \subseteq N, v(S)$ is the worth of coalition $S$, i.e., the members of coalition $S$ can obtain a total payoff of $v(S)$ by agreeing to cooperate.

We denote the set of all characteristic functions on player set $N$ by $\mathcal{G}^{N}$. For simplicity of notation and if no ambiguity appears, we write $v$ instead of $\langle N, v\rangle$. The subgame of $v$ with respect to a player set $T \subseteq N, T \neq \emptyset$, is the game $\left.v\right|_{T}$ defined as $\left.v\right|_{T}(S)=v(S)$, for all $S \subseteq T$. We denote the cardinality of a given set $A$ by $|A|$, along with lower case letters like $n=|N|, m=|M|, n_{k}=\left|N_{k}\right|, s=|S|, c=|C|, c^{\prime}=\left|C^{\prime}\right|$, and so on. For $K \subset \mathbb{N}$, we denote $\mathbb{R}^{K}$ as the $k$-dimensional vector space which elements $x \in \mathbb{R}^{K}$ have components $x_{i}, i \in K$. For every $x \in \mathbb{R}^{N}$ and $S \subseteq N$, we use the standard notation $x(S)=\sum_{i \in S} x_{i}$ and $x_{S}=\left\{x_{i}\right\}_{i \in S}$.

For game $v \in \mathcal{G}^{N}$, a vector $x \in \mathbb{R}^{N}$ may be considered as a payoff vector assigning 
a payoff $x_{i}$ to each player $i \in N$. A single-valued solution, called a value, is a mapping $\xi$ that assigns for every $N \subset \mathbb{N}$ and every $v \in \mathcal{G}^{N}$ a payoff vector $\xi(v) \in \mathbb{R}^{N}$. A value $\xi$ is efficient if $\sum_{i \in N} \xi_{i}(v)=v(N)$ for every $v \in \mathcal{G}^{N}$ and $N \subset \mathbb{N}$. The best-known efficient value is the Shapley value [11], given by

$$
S h_{i}(v)=\sum_{\{S \subseteq N \mid i \in S\}} \frac{(n-s) !(s-1) !}{n !}(v(S)-v(S \backslash\{i\})), \quad \text { for all } \quad i \in N .
$$

\subsection{Games with coalition structure}

A coalition structure on $N \subset \mathbb{N}$ is given by a partition $\mathcal{P}=\left\{N_{1}, \ldots, N_{m}\right\}$ of $N$. Elements of a partition will be called a priori unions. Let $\mathcal{C}^{N}$ denote the set of all coalition structures on $N$. A pair $\langle v, \mathcal{P}\rangle \in \mathcal{G}^{N} \times \mathcal{C}^{N}$ constitutes a game with coalition structure. A game with coalition structure represents situations in which a priori unions are formed. For partition $\mathcal{P}=\left\{N_{1}, \ldots, N_{m}\right\}$, we denote $M=\{1, \ldots, m\}$ and for every $i \in N$, we denote by $k(i)$ the index of the a priori union containing player $i$, so $k(i)$ is defined by the relation $i \in N_{k(i)}$. For any payoff vector $x \in \mathbb{R}^{N}$, let $x^{\mathcal{P}}=\left(x\left(N_{k}\right)\right)_{k \in M} \in \mathbb{R}^{M}$ be the corresponding vector of total payoffs to the a priori unions.

A value for games with coalition structures is a mapping $\xi$ that assigns for every $N$ and every $\langle v, \mathcal{P}\rangle \in \mathcal{G}^{N} \times \mathcal{C}^{N}$ a payoff vector $\xi(v, \mathcal{P}) \in \mathbb{R}^{N}$. One of the best-known values for games with coalition structures is the Owen value [10] that can be seen as a two-step procedure in which the Shapley value applies twice. ${ }^{1}$ First, for every a priori union the total payoff to the players within that union is determined by applying the Shapley value to the so-called quotient game being the game $v_{\mathcal{P}} \in \mathcal{G}^{M}, M=\{1, \ldots, m\}$, in which the unions act as individual players,

$$
v_{\mathcal{P}}(Q)=v\left(\cup_{k \in Q} N_{k}\right), \quad \text { for all } Q \subseteq M \text {. }
$$

So, the worth of a coalition $Q$ of a priori unions of $M$ in game $v_{\mathcal{P}}$ is the worth of the union of all coalitions in $Q$. The Shapley value of game $v_{\mathcal{P}}$ gives the total payoff of the Owen value to the a priori unions of the coalition structure. Second, the individual payoffs of the players within an a priori union are obtained by applying the Shapley value to a game on the players within the union. For every a priori union $k \in M$, this game $\bar{v}_{k} \in \mathcal{G}^{N_{k}}$ on player set $N_{k}$ is given by

$$
\bar{v}_{k}(S)=S h_{k}\left(\hat{v}_{S}\right), \quad S \subseteq N_{k},
$$

\footnotetext{
${ }^{1}$ See also van den Brink and van der Laan [2], in which Owen-type values for the class of games with coalition structures are given that determine the individual payoffs as the multiplicative product of two shares in the total payoff.
} 
where, for every $S \subseteq N_{k}$, the game $\hat{v}_{S} \in \mathcal{G}^{M}$ on the player set $M$ of a priori unions, is defined by

$$
\hat{v}_{S}(Q)=\left\{\begin{array}{ll}
v\left(\cup_{h \in Q} N_{h}\right), & k \notin Q, \\
v\left(\cup_{h \in Q \backslash\{k\}} N_{h} \cup S\right), & k \in Q,
\end{array} \quad \text { for all } Q \subseteq M .\right.
$$

So, for every $S \subseteq N_{k}$, the worth of subset $Q$ of $M$ in game $\hat{v}_{S}$ is the worth of the union of all coalitions in $Q$, except that coalition $N_{k}$ is replaced by $S \subseteq N_{k}$. Then the worth of coalition $S \subseteq N_{k}$ in $\bar{v}_{k}$ is the payoff that the Shapley value assigns to $k \in M$ in game $\hat{v}_{S}$. The Owen value assigns to player $i \in N$ the Shapley value of player $i$ in the game $\bar{v}_{k(i)}$, i.e.,

$$
O w_{i}(v, \mathcal{P})=S h_{i}\left(\bar{v}_{k(i)}\right), \quad \text { for all } i \in N \text {. }
$$

Notice that for every $k \in M$, the game $\hat{v}_{N_{k}}$ is equal to the quotient game $v_{\mathcal{P}}$. It is wellknown that the Owen value is efficient.

Another well-known solution for games with coalition structures is the AumannDrèze value [1] which assigns to every game $\langle v, \mathcal{P}\rangle \in \mathcal{G}^{N} \times \mathcal{C}^{N}$ the payoff vector

$$
A D_{i}(v, \mathcal{P})=S h_{i}\left(\left.v\right|_{N_{k(i)}}\right), \quad \text { for all } i \in N \text {. }
$$

The Aumann-Drèze value assigns to a player $i$ the Shapley payoff of player $i$ in the subgame on the coalition $N_{k}$ containing $i$. Notice that $\sum_{i \in N_{k}} A D_{i}(v, \mathcal{P})=v\left(N_{k}\right)$, and thus $\sum_{i \in N} A D_{i}(v, \mathcal{P})=\sum_{k \in M} v\left(N_{k}\right)$. Therefore the Aumann-Drèze value is not efficient. In fact, according to the Aumann-Drèze value it is assumed that every a priori union is a stand-alone coalition.

\subsection{Communication graph games}

For $N \subset \mathbb{N}$, a communication structure on $N$ is specified by a communication graph $\langle N, \Gamma\rangle$ with $\Gamma \subseteq \Gamma^{N}=\{\{i, j\} \mid i, j \in N, i \neq j\}$, i.e., $\Gamma$ is a collection of (unordered) pairs of nodes (players), where a pair $\{i, j\}$ represents a link between players $i, j \in N$, and $\left\langle N, \Gamma^{N}\right\rangle$ is the complete graph on $N$. Again, for simplicity of notation and if no ambiguity appears, we write graph $\Gamma$ instead of $\langle N, \Gamma\rangle$. Let $\mathcal{L}^{N}$ denote the set of all communication graphs on $N$. A pair $\langle v, \Gamma\rangle \in \mathcal{G}^{N} \times \mathcal{L}^{N}$ constitutes a game with (communication) graph structure or simply a graph game on $N$. For given $N$, the subgraph of a graph $\Gamma \in \mathcal{L}^{N}$ with respect to set $S \subseteq N, S \neq \emptyset$, is the graph $\left.\Gamma\right|_{S} \in \mathcal{L}^{S}$ defined by $\left.\Gamma\right|_{S}=\{\{i, j\} \in \Gamma \mid i, j \in S\}$. For ease of notation given digraph $\Gamma$ and link $\{i, j\} \in \Gamma$ the subgraph $\Gamma \backslash\{\{i, j\}\}$ we denote via $\left.\Gamma\right|_{-i j}$.

For a graph $\Gamma$ on $N$, a sequence of different nodes $\left(i_{1}, \ldots, i_{k}\right), k \geq 2$, is a path from $i_{1}$ to $i_{k}$, if for all $h=1, \ldots, k-1,\left\{i_{h}, i_{h+1}\right\} \in \Gamma$. A graph $\Gamma$ on a player set $N$ is connected, if for any two nodes in $N$ there exists a path in $\Gamma$ from one node to the other. 
For given graph $\Gamma$ on $N$, we say that the player set $S \subseteq N$ is connected, if the subgraph $\left.\Gamma\right|_{S}$ is connected. For graph $\Gamma$ on player set $N$ and $S \subseteq N$, a subset $T \subseteq S$ is a maximally connected subset or component of $S$ if (i) $\left.\Gamma\right|_{T}$ is connected, and (ii) for every $i \in S \backslash T$, the subgraph $\left.\Gamma\right|_{T \cup\{i\}}$ is not connected. For $\Gamma$ on $N$ and $S \subseteq N$, we denote by $S / \Gamma$ the set of all components of $S$, and by $(S / \Gamma)_{i}$ the component of $S$ containing $i \in S$. Notice that $S / \Gamma$ is a partition of $S$.

A value for communication graph games, a graph game value, is a mapping $\xi$ that for every $N \subset \mathbb{N}$ and every $\langle v, \Gamma\rangle \in \mathcal{G}^{N} \times \mathcal{L}^{N}$ assigns a payoff vector $\xi(v, \Gamma) \in \mathbb{R}^{N}$. A graph game value $\xi$ is component efficient if for any $\langle v, \Gamma\rangle \in \mathcal{G}^{N} \times \mathcal{L}^{N}, \sum_{i \in C} \xi_{i}(v, \Gamma)=v(C)$ for every $C \in N / \Gamma$. A well-known component efficient graph game value is the Myerson value. Following Myerson [9], we assume that in a communication graph game $\langle v, \Gamma\rangle$ only connected coalitions are able to cooperate and to realise their worths. A non-connected coalition $S$ can only realise the sum of the worths of its components in $S / \Gamma$. This yields the restricted game $v^{\Gamma} \in \mathcal{G}^{N}$ defined by

$$
v^{\Gamma}(S)=\sum_{T \in S / \Gamma} v(T), \quad \text { for all } S \subseteq N
$$

Then the Myerson value for communication graph games is the graph game value $\mu$ that assigns to every communication graph game $\langle v, \Gamma\rangle$ the Shapley value of its restricted game $v^{\Gamma}$, i.e.,

$$
\mu(v, \Gamma)=S h_{i}\left(v^{\Gamma}\right)
$$

It is well-known that the Myerson value is the unique graph game value that is component efficient and satisfies the so-called Myerson fairness axiom. A graph game value $\xi$ is fair if for every graph game $\langle v, \Gamma\rangle$ on any player set $N$, for every $\{h, k\} \in \Gamma$, $\xi_{h}(v, \Gamma)-\xi_{h}\left(v,\left.\Gamma\right|_{-h k}\right)=\xi_{k}(v, \Gamma)-\xi_{k}\left(v,\left.\Gamma\right|_{-h k}\right)$.

\section{Games with two-level communication structures}

We now consider situations in which the players are partitioned into a coalition structure $\mathcal{P}$ and are linked to each other by communication graphs. First, there is a communication graph $\Gamma_{M}$ between the a priori unions $M$ in the partition $\mathcal{P}$. Second, for each a priori union $N_{k}, k \in M$, there is a communication graph $\Gamma_{k}$ between the players in $N_{k}$. Given $\mathcal{P} \in \mathcal{C}^{N}$, a two-level communication structure on $N$ is given by the tuple $\Gamma_{\mathcal{P}}=\left\langle\Gamma_{M},\left\{\Gamma_{k}\right\}_{k \in M}\right\rangle$.

For $N \subset \mathbb{N}$ and $\mathcal{P} \in \mathcal{C}^{N}$ let $\mathcal{L}_{\mathcal{P}}^{N}$ be the set of all two-level communication structures on $N$ with fixed $\mathcal{P}$ and let $\mathcal{L}_{\mathcal{C}}^{N}=\bigcup_{\mathcal{P} \in \mathcal{C}^{N}} \mathcal{L}_{\mathcal{P}}^{N}$ be the set of all two-level graph structures on $N$. A tuple $\left\langle v, \Gamma_{\mathcal{P}}\right\rangle \in \mathcal{G}^{N} \times \mathcal{L}_{\mathcal{C}}^{N}$ constitutes a game with two-level communication structure 
or simply two-level graph game on $N$. A value for games with two-level communication structure, a two-level graph game value, is a mapping $\xi$ that assigns for every $N \subset \mathbb{N}$ and every two-level graph game $\left\langle v, \Gamma_{\mathcal{P}}\right\rangle \in \mathcal{G}^{N} \times \mathcal{L}_{\mathcal{C}}^{N}$ a payoff vector $\xi\left(v, \Gamma_{\mathcal{P}}\right) \in \mathbb{R}^{N}$.

We now state several axioms that can be satisfied by solutions for games with twolevel communication structures. The first three axioms are generalizations of axioms used to characterize the Myerson value on the class of communication graph games. First, quotient component efficiency states that on the level of the a priori unions (in the sequel shortly to be called the upper level), the total payoff of the players in the a priori unions of a component $K \in M / \Gamma_{M}$ is equal to the worth of the unions in the component in the quotient game $v_{\mathcal{P}}$ on $M$.

Axiom 3.1 (Quotient Component Efficiency (QCE)) For any player set $N \subset \mathbb{N}$, for every $\left\langle v, \Gamma_{\mathcal{P}}\right\rangle \in \mathcal{G}^{N} \times \mathcal{L}_{\mathcal{C}}^{N}$, it holds

$$
\sum_{k \in K} \sum_{i \in N_{k}} \xi_{i}\left(v, \Gamma_{\mathcal{P}}\right)=v_{\mathcal{P}}(K), \quad \text { for every } K \in M / \Gamma_{M}
$$

Quotient component efficiency requires the same as the axiom 'component efficiency in quotient' used in Khmelnitskaya [7] for every non-singleton component $K \in M / \Gamma_{M}$ and every singleton component $K=\{k\}, k \in M$, for which the corresponding graph $\Gamma_{k}$ is connected. When $K \in M / \Gamma_{M}$ is a singleton component $\{k\}$ with $\Gamma_{k}$ not connected, then the 'component efficiency in quotient' of [7] requires that the total payoff to the players in $N_{k}$ is equal to $\sum_{C \in N_{k} / \Gamma_{k}} v(C)$, whereas quotient component efficiency still requires that the total payoff to the players in $N_{k}$ is equal to $v_{\mathcal{P}}(\{k\})=v\left(N_{k}\right)$. So, in this case, for the union $N_{k}$, [7] requires component efficiency with respect to the within union communication graph $\Gamma_{k}$, whereas quotient component efficiency requires efficiency within $N_{k}$. Notice that the Myerson value of the quotient game $v_{\mathcal{P}}$ with respect to $\Gamma_{M}$ yields payoff $v\left(N_{k}\right)$ to union $k$ when $\{k\}$ is a singleton component in $\Gamma_{M}$.

The next axiom applies the well-known Myerson fairness axiom between unions, i.e., it applies fairness on the upper level with respect to the quotient game. If a link $\{k, h\} \in \Gamma_{M}$ is removed from the graph $\Gamma_{M}$ on the upper level, then the change in the total payoff to a priori union $N_{k}$ is equal to the change in the total payoff to a priori union $N_{h}$. For $\Gamma_{\mathcal{P}}=\left\langle\Gamma_{M},\left\{\Gamma_{k}\right\}_{k \in M}\right\rangle$ and link $\{k, h\} \in \Gamma_{M}$, we denote by $\left.\Gamma_{\mathcal{P}}\right|_{-k h}$ the tuple $\left\langle\left.\Gamma_{M}\right|_{-k h},\left\{\Gamma_{k}\right\}_{k \in M}\right\rangle$.

Axiom 3.2 (Quotient Fairness (QF)) For any player set $N \subset \mathbb{N}$, for every $\left\langle v, \Gamma_{\mathcal{P}}\right\rangle \in$ $\mathcal{G}^{N} \times \mathcal{L}_{\mathcal{C}}^{N}$, and every $\{k, h\} \in \Gamma_{M}$, it holds

$$
\sum_{i \in N_{k}}\left(\xi_{i}\left(v, \Gamma_{\mathcal{P}}\right)-\xi_{i}\left(v,\left.\Gamma_{\mathcal{P}}\right|_{-k h}\right)\right)=\sum_{i \in N_{h}}\left(\xi_{i}\left(v, \Gamma_{\mathcal{P}}\right)-\xi_{i}\left(v,\left.\Gamma_{\mathcal{P}}\right|_{-k h}\right)\right)
$$


Quotient fairness is similar to 'fairness in the quotient' used by Vázquez-Brage et al. [12], but within the different framework of only one communication graph between all players. The quotient fairness axiom is weaker than the 'between block fairness' of Kongo [8] which not only requires quotient fairness, but also that when in $\Gamma_{M}$ a link between two unions is deleted, within each of the two unions the change in payoff is the same for all players within that union.

In the next section it will be shown that the axioms above uniquely determine the total payoff to every a priori union $N_{k}$ in the coalition structure $\mathcal{P}$, similar as in Myerson [9] for a one-level communication graph. In fact, it follows that the total payoff to coalition $N_{k}$ is equal to the Myerson payoff to union $k \in M$ of the quotient game $v_{\mathcal{P}}$ with respect to the upper level communication graph $\Gamma_{M}$ between the unions.

The next two axioms will determine for every $k \in M$ the distribution of the total payoff assigned to coalition $N_{k}$ amongst the players in $N_{k}$. The first one applies the Myerson fairness axiom within the unions, i.e., if a link $\{i, j\} \in \Gamma_{k}$ is removed from the communication graph $\Gamma_{k}$ within the union $N_{k}$, then the change of payoff to player $i$ is equal to the change of payoff to player $j$. For $\Gamma_{\mathcal{P}}=\left\langle\Gamma_{M},\left\{\Gamma_{h}\right\}_{h \in M}\right\rangle$ and link $\{i, j\} \in \Gamma_{k}, k \in M$, we denote by $\left.\Gamma_{\mathcal{P}}\right|_{-i j} ^{k}$ the tuple $\left\langle\Gamma_{M},\left\{\widehat{\Gamma}_{h}\right\}_{h \in M}\right\rangle$ where $\widehat{\Gamma}_{h}=\Gamma_{h}$ for $h \neq k$, and $\widehat{\Gamma}_{k}=\left.\Gamma_{k}\right|_{-i j}$.

Axiom 3.3 (Union Fairness (UF)) For any player set $N \subset \mathbb{N}$, for every $\left\langle v, \Gamma_{\mathcal{P}}\right\rangle \in$ $\mathcal{G}^{N} \times \mathcal{L}_{\mathcal{C}}^{N}, k \in M$, and $\{i, j\} \in \Gamma_{k}$, it holds

$$
\xi_{i}\left(v, \Gamma_{\mathcal{P}}\right)-\xi_{i}\left(v,\left.\Gamma_{\mathcal{P}}\right|_{-i j} ^{k}\right)=\xi_{j}\left(v, \Gamma_{\mathcal{P}}\right)-\xi_{j}\left(v,\left.\Gamma_{\mathcal{P}}\right|_{-i j} ^{k}\right)
$$

The union fairness axiom is the same as the 'within block fairness' axiom in Kongo [8]. Quotient fairness requires Myerson fairness on the upper level, while union fairness requires Myerson fairness on the lower level. Also in the ' $(m+1)$-tuple of deletion link axioms' used in Khmelnitskaya [7], Myerson fairness can be applied both on the upper level and the lower level. In this case the requirement of $(m+1)$-tuple of deletion link axioms in [7] is similar to the total requirement of both quotient fairness and union fairness axioms.

As it was already mentioned before, the total payoff assigned to the players in $N_{k}$ in the quotient game on the upper level has to be fully distributed over the players in $N_{k}$ in the game within the union, also when the communication graph $\Gamma_{k}$ partitions the union $N_{k}$ into several components. So, within an a priori union $N_{k}$ we have efficiency in the sense that the total payoff assigned to $N_{k}$ is distributed and thus within $N_{k}$ the component efficiency axiom does not hold. The last axiom determines the distribution of the total payoff to $N_{k}$ among the several components of $N_{k}$ in the communication graph $\Gamma_{k}$. For some $k \in M$ and component $C \in N_{k} / \Gamma_{k}$, let $v_{C}^{k}$ denote the subgame $\left.v\right|_{\left(N \backslash N_{k}\right) \cup C}$ of $v$ with respect to the coalition $\left(N \backslash N_{k}\right) \cup C$. Further, let $\mathcal{P}_{C}^{k}$ denote the partition on $\left(N \backslash N_{k}\right) \cup C$ consisting of union $C$ and all unions $N_{h}$ in $\mathcal{P}, h \neq k$, and let $\Gamma_{\mathcal{P}_{C}^{k}}=\left\langle\Gamma_{M},\left\{\widetilde{\Gamma}_{h}\right\}_{h \in M}\right\rangle$ with $\widetilde{\Gamma}_{k}=\left.\Gamma_{k}\right|_{C}$ 
and $\widetilde{\Gamma}_{h}=\Gamma_{h}$ for all $h \in M \backslash\{k\}$, denote the two-level communication structure that is obtained from $\left\langle\Gamma_{M},\left\{\Gamma_{h}\right\}_{h \in M}\right\rangle$ by replacing the communication graph $\Gamma_{k}$ by its restriction on $C \subset N_{k}{ }^{2}$ This axiom applies the component balancedness axiom for communication graph games, introduced recently in van den Brink, Khmelnitskaya, and van der Laan [3], to graph games within the unions.

Axiom 3.4 (Union Component Balancedness (UCB)) For any player set $N \subset \mathbb{N}$, for every $\left\langle v, \Gamma_{\mathcal{P}}\right\rangle \in \mathcal{G}^{N} \times \mathcal{L}_{\mathcal{C}}^{N}, k \in M$, and component $C \in N_{k} / \Gamma_{k}$, it holds

$$
\frac{1}{c} \sum_{i \in C}\left(\xi_{i}\left(v, \Gamma_{\mathcal{P}}\right)-\xi_{i}\left(v_{C}^{k}, \Gamma_{\mathcal{P}_{C}^{k}}^{k}\right)\right)=\frac{1}{n_{k}} \sum_{i \in N_{k}}\left(\xi_{i}\left(v, \Gamma_{\mathcal{P}}\right)-\xi_{i}\left(v_{\left(N_{k} / \Gamma_{k}\right)_{i}}^{k}, \Gamma_{\mathcal{P}_{\left(N_{k} / \Gamma_{k}\right)_{i}}^{k}}\right)\right) .
$$

Notice that this axiom only states a requirement for the distribution of the total payoff within a union $N_{k}$ when $N_{k}$ consists of multiple components with respect to the internal communication graph $\Gamma_{k}$, otherwise the requirement reduces to an identity. Since

$$
\sum_{i \in N_{k}} \xi_{i}\left(v_{\left(N_{k} / \Gamma_{k}\right)_{i}}^{k}, \Gamma_{\mathcal{P}_{\left(N_{k} / \Gamma_{k}\right)_{i}}^{k}}\right)=\sum_{H \in N_{k} / \Gamma_{k}} \sum_{i \in H} \xi_{i}\left(v_{H}^{k}, \Gamma_{\mathcal{P}_{H}^{k}}\right),
$$

it follows that for some component $C \in N_{k} / \Gamma_{k}$ union component balancedness also can be written as

$$
\sum_{i \in C}\left(\xi_{i}\left(v, \Gamma_{\mathcal{P}}\right)-\xi_{i}\left(v_{C}^{k}, \Gamma_{\mathcal{P}_{C}^{k}}\right)\right)=\frac{c}{n_{k}}\left(\sum_{i \in N_{k}} \xi_{i}\left(v, \Gamma_{\mathcal{P}}\right)-\sum_{H \in N_{k} / \Gamma_{k}} \sum_{i \in H} \xi_{i}\left(v_{H}^{k}, \Gamma_{\mathcal{P}_{H}^{k}}\right)\right) .
$$

Since for $i \in H \in N_{k} / \Gamma_{k}, \xi_{i}\left(v_{H}^{k}, \Gamma_{\mathcal{P}_{H}^{k}}\right)$ is the payoff to player $i$ when the component $H \in N_{k} / \Gamma_{k}$ containing $i$ replaces $N_{k}$ in the game between the unions, union component balancedness means that the excess (positive or negative), realized by the players of $N_{k}$ when they all cooperate together in the game between the unions (instead of the cooperation within $N_{k}$ being restricted to players within one component of $N_{k} / \Gamma_{k}$ ) is distributed to the components in proportion to the number of players in the components. Union component balancedness is equivalent to saying that for any two components $C, C^{\prime} \in N_{k} / \Gamma_{k}$,

$$
\left.\frac{1}{c} \sum_{i \in C}\left(\xi_{i}\left(v, \Gamma_{\mathcal{P}}\right)-\xi_{i}\left(v_{C}^{k}, \Gamma_{\mathcal{P}_{C}^{k}}^{k}\right)\right)\right)=\frac{1}{c^{\prime}} \sum_{i \in C^{\prime}}\left(\xi_{i}\left(v, \Gamma_{\mathcal{P}}\right)-\xi_{i}\left(v_{C^{\prime}}^{k}, \Gamma_{\mathcal{P}_{C^{\prime}}^{k}}\right)\right),
$$

meaning that considering only the players in component $C$ in $\Gamma_{k}$ instead of all players in $N_{k}$, the change in the average payoff of the players in this component is the same as the change in the average payoff of the players in any other component $C^{\prime}$ resulting from considering only the players in that component $C^{\prime}$ in $\Gamma_{k}$. Therefore, the last equality provides an alternative representation of the union component balancedness axiom.

\footnotetext{
${ }^{2}$ Note that in this axiom we consider games with two-level communication structures where the player set $N$ is replaced by $\left(N \backslash N_{k}\right) \cup C$. To be precise we therefore need to write such a game as a triple $\left(N, v, \Gamma_{\mathcal{P}}\right)$, respectively $\left(\left(N \backslash N_{k}\right) \cup C, v_{C}^{k}, \Gamma_{\mathcal{P}_{C}^{k}}\right)$. Since the player set is clear from the context, we ignore the player set in the notation of a game.
} 


\section{An Owen-type value for two-level graph games}

In this section we first show that there exists a two-level graph game value that satisfies the four axioms. After that we show that this solution is characterized by the four axioms, i.e., it is the unique two-level graph game value satisfying these axioms.

Analogously to the Owen value for games with coalition structures, we introduce an Owen-type value for the class of games with two-level communication structures. First, for every $k \in M$ and $S \subseteq N_{k}$, recall the game $\hat{v}_{S} \in \mathcal{G}^{M}$ on the player set $M$ of a priori unions defined by (2.2), where the worth of a coalition $Q$ of a priori unions of $M$ equals the worth of the union of all unions in $Q$, except that union $N_{k}$ is replaced by $S \subseteq N_{k}$. We now take into account the communication graph $\Gamma_{M}$ between the a priori unions. Instead of the game $\bar{v}_{k} \in \mathcal{G}^{N_{k}}$ on player set $N_{k}$ given by (2.1), we now define the game $\widetilde{v}_{k} \in \mathcal{G}^{N_{k}}$ by taking the Myerson value of $\hat{v}$ with respect to $\Gamma_{M}$ instead of the Shapley value of $\hat{v}$. So,

$$
\widetilde{v}_{k}(S)=\mu_{k}\left(\hat{v}_{S}, \Gamma_{M}\right)=S h_{k}\left(\hat{v}_{S}^{\Gamma_{M}}\right), \quad \text { for all } S \subseteq N_{k} .
$$

Notice that $\widetilde{v}_{k}\left(N_{k}\right)=S h_{k}\left(\hat{v}_{N_{k}}^{\Gamma_{M}}\right)=S h_{k}\left(v_{\mathcal{P}}^{\Gamma_{M}}\right)$, i.e., the worth of $N_{k}$ in the game $\widetilde{v}_{k}$ is equal to the Myerson value of $k \in M$ (representing union $N_{k}$ ) in the quotient game with respect to the communication graph $\Gamma_{M}$. Next, recall again from Subsection 2.2 that without communication graphs, the Owen value of a player $i \in N_{k}$ is the Shapley payoff to player $i$ in the game $\bar{v}_{k} \in \mathcal{G}^{N_{k}}$. Taking into account the communication graph $\Gamma_{k}$ within $N_{k}$, we take for player $i \in N_{k}$ its Shapley payoff in a modification of the Myerson restricted game $\widetilde{v}_{k}^{\Gamma_{k}}$ of the game $\widetilde{v}_{k} \in \mathcal{G}^{N_{k}}$. The modification concerns the worth of the coalition $N_{k}$ itself, for which we take its own worth $\widetilde{v}_{k}\left(N_{k}\right)$ instead of the sum of the worths of components $\sum_{C \in N_{k} / \Gamma_{k}} \widetilde{v}_{k}(C)$. This is because the players in $N_{k}$ have to distribute the total payoff assigned to a priori union $N_{k}$ in the restricted quotient game. The value constructed in this way is denoted by $\psi$, so,

$$
\psi_{i}\left(v, \Gamma_{\mathcal{P}}\right)=S h_{i}\left(\tilde{\tilde{v}}_{k(i)}\right), \quad \text { for all } i \in N, N \in \mathbb{N}
$$

where for all $k \in M, \tilde{\tilde{v}}_{k} \in \mathcal{G}^{N_{k}}$ is defined by

$$
\tilde{\tilde{v}}_{k}(S)= \begin{cases}\widetilde{v}_{k}^{\Gamma_{k}}(S), & S \varsubsetneqq N_{k}, \\ \widetilde{v}_{k}\left(N_{k}\right)=S h_{k}\left(v_{\mathcal{P}}^{\Gamma_{M}}\right), & S=N_{k} .\end{cases}
$$

Analogously to the Owen value, the value $\psi$ can be seen as a two-step procedure in which first every coalition gets its Shapley value of the Myerson restriction of the quotient game with respect to communication graph $\Gamma_{M}$, and second every player $i$ in a priori union $N_{k}$ gets its Shapley payoff in the within a priori union game $\tilde{\tilde{v}}_{k} \in \mathcal{G}^{N_{k}}$. We now have the following theorem.

Theorem 4.1 The two-level graph game value $\psi$ satisfies QCE, QF, UF and UCB. 


\section{Proof.}

QCE. First,

$$
\sum_{i \in N_{k}} \psi_{i}\left(v, \Gamma_{\mathcal{P}}\right)=\sum_{i \in N_{k}} S h_{i}\left(\tilde{\tilde{v}}_{k(i)}\right)=S h_{k}\left(v_{\mathcal{P}}^{\Gamma_{M}}\right)=\mu_{k}\left(v_{\mathcal{P}}, \Gamma_{M}\right)
$$

where the first equality follows by definition of $\psi$, the second equality follows from efficiency of the Shapley value, and the third equality follows from the definition of the Myerson value $\mu$. Thus, we have

$$
\sum_{k \in K} \sum_{i \in N_{k}} \psi_{i}\left(v, \Gamma_{\mathcal{P}}\right)=\sum_{k \in K} \mu_{k}\left(v_{\mathcal{P}}, \Gamma_{M}\right)=v_{\mathcal{P}}(K)
$$

where the first equality follows from (4.3) and the second equality follows from component efficiency of $\mu$.

QF. We have

$$
\begin{aligned}
\sum_{i \in N_{k}} \psi_{i}\left(v, \Gamma_{\mathcal{P}}\right)- & \sum_{i \in N_{k}} \psi_{i}\left(v,\left.\Gamma_{\mathcal{P}}\right|_{-k h}\right)= \\
& \mu_{k}\left(v_{\mathcal{P}}, \Gamma_{M}\right)-\mu_{k}\left(v_{\mathcal{P}},\left.\Gamma_{M}\right|_{-k h}\right)=\mu_{h}\left(v_{\mathcal{P}}, \Gamma_{M}\right)-\mu_{h}\left(v_{\mathcal{P}},\left.\Gamma_{M}\right|_{-k h}\right)= \\
& \sum_{i \in N_{h}} \psi_{i}\left(v, \Gamma_{\mathcal{P}}\right)-\sum_{i \in N_{h}} \psi_{i}\left(v,\left.\Gamma_{\mathcal{P}}\right|_{-k h}\right)
\end{aligned}
$$

where the first and third equality follow from (4.3), and the second equality follows by fairness of $\mu$.

UF. By definition

$$
\tilde{\tilde{v}}_{k(i)}=\tilde{v}_{k(i)}^{\Gamma_{k(i)}}+w
$$

where $w \in \mathcal{G}^{N_{k(i)}}$ is given by

$$
w(S)= \begin{cases}0, & S \varsubsetneqq N_{k(i)}, \\ S h_{k}\left(v_{\mathcal{P}}^{\Gamma_{M}}\right)-\tilde{v}_{k(i)}^{\Gamma_{k(i)}}\left(N_{k(i)}\right), & S=N_{k(i)},\end{cases}
$$

i.e., game $\tilde{\tilde{v}}_{k(i)}$ is obtained by adding $\left(S h_{k}\left(v_{\mathcal{P}}^{\Gamma_{M}}\right)-\tilde{v}_{k(i)}^{\Gamma_{k(i)}}\left(N_{k(i)}\right)\right)$ times the unanimity game ${ }^{3}$ of $N_{k(i)}$ to the game $\tilde{v}_{k(i)}^{\Gamma_{k(i)}}$. From this it follows that

$$
\psi_{i}\left(v, \Gamma_{\mathcal{P}}\right)=S h_{i}\left(\tilde{\tilde{v}}_{k(i)}\right)=S h_{i}\left(\tilde{v}_{k(i)}^{\Gamma_{k(i)}}\right)+\frac{S h_{k}\left(v_{\mathcal{P}}^{\Gamma_{M}}\right)-\tilde{v}_{k(i)}^{\Gamma_{k(i)}}\left(N_{k(i)}\right)}{n_{k(i)}}=
$$

\footnotetext{
${ }^{3}$ It is well known [11] that the collection of unanimity games $\left\{u_{T}\right\}_{\substack{T \subseteq N \\ T \neq \emptyset}}$, defined as $u_{T}(S)=1$, if $T \subseteq S$, and $u_{T}(S)=0$ otherwise, create a basis in $\mathcal{G}^{N}$.
} 


$$
\mu_{i}\left(\tilde{v}_{k(i)}, \Gamma_{k(i)}\right)+\frac{\mu_{k(i)}\left(v_{\mathcal{P}}, \Gamma_{M}\right)-\sum_{C \in N_{k(i)} / \Gamma_{k(i)}} v(C)}{n_{k(i)}},
$$

where the first equality follows by definition of the value $\psi$, the second equality follows from additivity of the Shapley value and (4.4), and the third equality follows by definition of $\mu$ and $\tilde{v}_{k(i)}^{\Gamma_{k(i)}}$. Hence,

$$
\begin{aligned}
\psi_{i}\left(v, \Gamma_{\mathcal{P}}\right)-\psi_{i}\left(v,\left.\Gamma_{\mathcal{P}}\right|_{-i j} ^{k(i)}\right)=\mu_{i}\left(\tilde{v}_{k(i)}, \Gamma_{k(i)}\right)-\mu_{i}\left(\tilde{v}_{k(i)},\left.\Gamma_{k(i)}\right|_{-i j}\right)+ & \\
& \frac{\mu_{k(i)}\left(v_{\mathcal{P}}, \Gamma_{M}\right)-\sum_{C \in N_{k(i)} / \Gamma_{k(i)}} v(C)}{n_{k(i)}}-\frac{\mu_{k(i)}\left(v_{\mathcal{P}}, \Gamma_{M}\right)-\sum_{C \in N_{k(i)} /\left.\Gamma_{k(i)}\right|_{-i j}} v(C)}{n_{k(i)}}= \\
& \mu_{j}\left(\tilde{v}_{k(i)}, \Gamma_{k(i)}\right)-\mu_{j}\left(\tilde{v}_{k(i)},\left.\Gamma_{k(i)}\right|_{-i j}\right)+ \\
& \frac{\mu_{k(i)}\left(v_{\mathcal{P}}, \Gamma_{M}\right)-\sum_{C \in N_{k(i)} / \Gamma_{k(i)}} v(C)}{n_{k(i)}}-\frac{\mu_{k(i)}\left(v_{\mathcal{P}}, \Gamma_{M}\right)-\sum_{C \in N_{k(i)} /\left.\Gamma_{k(i)}\right|_{-i j}} v(C)}{n_{k(i)}}= \\
& \psi_{j}\left(v, \Gamma_{\mathcal{P}}\right)-\psi_{j}\left(v,\left.\Gamma_{\mathcal{P}}\right|_{-i j} ^{k(i)}\right),
\end{aligned}
$$

where the first and third equality follow from (4.5), and the second equality follows by fairness of $\mu$.

UCB. By (4.5), we obtain for every $C \in N_{k} / \Gamma_{k}$ that

$$
\sum_{i \in C} \psi_{i}\left(v, \Gamma_{\mathcal{P}}\right)=\sum_{i \in C} \mu_{i}\left(\tilde{v}_{k}, \Gamma_{k}\right)+\frac{c}{n_{k}}\left(\mu_{k}\left(v_{\mathcal{P}}, \Gamma_{M}\right)-\sum_{H \in N_{k} / \Gamma_{k}} v(H)\right) .
$$

Further,

$$
\sum_{i \in C} \psi_{i}\left(v_{C}^{k}, \Gamma_{\mathcal{P}_{C}^{k}}\right)=\tilde{\tilde{v}}_{C}^{k}(C)=\tilde{v}_{C}^{k}(C)=\sum_{i \in C} \mu_{i}\left(\tilde{v}_{k}, \Gamma_{k}\right)
$$

where the first and second equality follow from the definition of $\psi$, efficiency of the Shapley value and $C$ being the only component in $\widetilde{\Gamma}_{k}$, and the third equality follows from component efficiency of $\mu$. Thus

$$
\sum_{i \in C}\left(\psi_{i}\left(v, \Gamma_{\mathcal{P}}\right)-\psi_{i}\left(v_{C}^{k}, \Gamma_{\mathcal{P}_{C}^{k}}\right)\right)=\frac{c}{n_{k}}\left(\mu_{k}\left(v_{\mathcal{P}}, \Gamma_{M}\right)-\sum_{H \in N_{k} / \Gamma_{k}} v(H)\right) .
$$

Similarly, we can derive

$$
\left.\sum_{i \in N_{k}}\left(\psi_{i}\left(v, \Gamma_{\mathcal{P}}\right)-\psi_{i}\left(v_{\left(N_{k} / \Gamma_{k}\right)_{i}}^{k}, \Gamma_{\mathcal{P}_{\left(N_{k} / \Gamma_{k}\right)_{i}}^{k}}\right)\right)=\mu_{k}\left(v_{\mathcal{P}}, \Gamma_{M}\right)-\sum_{H \in N_{k} / \Gamma_{k}} v(H)\right) .
$$


Hence it follows that

$$
\frac{1}{c} \sum_{i \in C}\left(\psi_{i}\left(v, \Gamma_{\mathcal{P}}\right)-\psi_{i}\left(v_{C}^{k}, \Gamma_{\mathcal{P}_{C}^{k}}\right)\right)=\frac{1}{n_{k}} \sum_{i \in N_{k}}\left(\psi_{i}\left(v, \Gamma_{\mathcal{P}}\right)-\psi_{i}\left(v_{\left(N_{k} / \Gamma_{k}\right)_{i}}^{k}, \Gamma_{\mathcal{P}_{\left(N_{k} / \Gamma_{k}\right)_{i}}^{k}}\right)\right),
$$

showing that $\psi$ satisfies UCB.

Remark Note that (4.5) gives an alternative definition of the value $\psi$ assigning to every graph game its Myerson value and distributing the difference between the worth of the grand coalition $N$ and the sum of the worths of all components equally over all players. In this sense $\psi$ can be seen as combining elements of the Myerson value and equal division solution. This idea is similar to Kamijo [6] who introduced a solution for games in coalition structure that allocates to every player its Shapley value in the game restricted to its own union and distributes the excess of the Shapley value of its union in the (quotient) game between the unions over the worth of this union equally among the players in this union.

The next theorem characterizes the value $\psi$ as the unique solution satisfying the four axioms.

Theorem 4.2 There is a unique two-level graph game value $\xi$ satisfying $Q C E, Q F$, UF and $U C B$.

Proof. By Theorem 4.1 we only need to show uniqueness. Let $\mathcal{P}=\left\{N_{1}, \ldots, N_{m}\right\} \in \mathcal{C}^{N}$ and $\left(v, \Gamma_{\mathcal{P}}\right) \in \mathcal{G}^{N} \times \mathcal{L}_{\mathcal{C}}^{N}$ with $\Gamma_{\mathcal{P}}=\left\langle\Gamma_{M},\left\{\Gamma_{h}\right\}_{h \in M}\right\rangle$. For a solution $\xi$, we denote $\xi^{k}\left(v, \Gamma_{\mathcal{P}}\right)=$ $\sum_{i \in N_{k}} \xi_{i}\left(v, \Gamma_{\mathcal{P}}\right)$ as the total payoff to the players in the union $N_{k}, k=1, \ldots, m$. Suppose that solution $\xi$ satisfies the four axioms. We determine the individual payoffs in three steps.

Step 1. We determine the 'union payoffs' in the game $\left(v, \Gamma_{\mathcal{P}}\right) \in \mathcal{G}^{N} \times \mathcal{L}_{\mathcal{C}}^{N}$ with $\Gamma_{\mathcal{P}}=$ $\left\langle\Gamma_{M},\left\{\Gamma_{h}\right\}_{h \in M}\right\rangle$ by induction on the number of links in $\Gamma_{M}$ in a similar way as uniqueness of the Myerson value for one-level graph games is shown in Myerson [9]. When $\left|\Gamma_{M}\right|=0$ then, for all $k \in M$, the set of neighboring unions $\{h \in M \mid\{h, k\} \in \Gamma\}=\emptyset$, and thus $\xi^{k}\left(v, \Gamma_{\mathcal{P}}\right)=v_{\mathcal{P}}\left(N_{k}\right)=v\left(N_{k}\right)$ by QCE.

Proceeding by induction, assume that the values $\xi^{k}\left(v, \Gamma_{\mathcal{P}}^{\prime}\right)$ have been determined whenever $\Gamma_{\mathcal{P}}^{\prime}=\left\langle\Gamma^{\prime},\left\{\Gamma_{h}\right\}_{h \in M}\right\rangle$ for every $\Gamma^{\prime}$ with $\left|\Gamma^{\prime}\right|<\left|\Gamma_{M}\right|$. Let $Q \in M / \Gamma_{M}$ be a component in $\left\langle M, \Gamma_{M}\right\rangle$. If $Q \subseteq M$ is a singleton set $\{k\}$, then it follows from QCE that $\xi^{k}\left(v, \Gamma_{\mathcal{P}}\right)=v\left(N_{k}\right)$. If $q=|Q| \geq 2$, then there exists a spanning tree $\left.\widetilde{\Gamma} \subseteq \Gamma_{M}\right|_{Q}$ on $Q$, i.e., $\langle Q, \widetilde{\Gamma}\rangle$ is connected and $\langle Q, \widetilde{\Gamma} \backslash\{k, h\}\rangle$ is not connected for all $\{k, h\} \in \widetilde{\Gamma}$. So, the number of links in $\widetilde{\Gamma}$ is $q-1$. By QF, for all $\{k, h\} \in \widetilde{\Gamma}$ it holds that

$$
\xi^{k}\left(v, \Gamma_{\mathcal{P}}\right)-\xi^{k}\left(v,\left.\Gamma_{\mathcal{P}}\right|_{-k h}\right)=\xi^{h}\left(v, \Gamma_{\mathcal{P}}\right)-\xi^{h}\left(v,\left.\Gamma_{\mathcal{P}}\right|_{-k h}\right) .
$$


Moreover, by QCE it holds that

$$
\sum_{k \in Q} \xi^{k}\left(v, \Gamma_{\mathcal{P}}\right)=v_{\mathcal{P}}(K)
$$

Since $\left|\Gamma_{M} \backslash\{h, k\}\right|=\left|\Gamma_{M}\right|-1$, it follows by the induction hypothesis that all the values $\xi^{k}\left(v,\left.\Gamma_{\mathcal{P}}\right|_{-k h}\right), \quad\{k, h\} \in \widetilde{\Gamma}$, have been determined, and thus (4.6) and (4.7) yield $q$ linear equations in the $q$ unknown payoffs $\xi^{k}\left(v, \Gamma_{\mathcal{P}}\right), k \in Q$. Since these equations are linearly independent, for every $Q \in M / \Gamma$, all payoffs $\xi^{k}\left(v, \Gamma_{\mathcal{P}}\right), k \in Q$, are uniquely determined. ${ }^{4}$

Step 2. Second, similarly as in Step 1, we determine for every $k \in M$, for every subset $C \subset N_{k}$ the 'union payoffs' in the game $\left(v_{C}, \Gamma_{\mathcal{P}_{C}^{k}}\right)$, where $v_{C}^{k}$ denotes the subgame $\left.v\right|_{\left(N \backslash N_{k}\right) \cup C}$ of $v$ with respect to the coalition $\left(N \backslash N_{k}\right) \cup C$, and $\Gamma_{\mathcal{P}_{C}^{k}}$ denotes the two-level communication structure $\left\langle\Gamma_{M},\left\{\Gamma_{h}\right\}_{h \in M}\right\rangle$, where $\Gamma_{M}$ is the communication graph on the partition $\left(\mathcal{P} \backslash\left\{N_{k}\right\}\right) \cup\{C\}$ (where the 'position' of $N_{k}$ is taken over by $C$ ) and with the communication graph $\Gamma_{k}$ replaced by its restriction on $C \subset N_{k}$. Note that now, for $k \in M$, the union payoff $\xi^{k}\left(v_{C}^{k}, \Gamma_{\mathcal{P}_{C}^{k}}\right)$ is the total payoff to the players in $C \subset N_{k}$ in the game $\left(v_{C}^{k}, \Gamma_{\mathcal{P}_{C}^{k}}\right)$.

Step 3. Third, we determine the individual payoffs in every coalition $N_{k}, k \in M$. Take some $k \in M$. If $\left|\Gamma_{k}\right|=0$ then $\{i\} \in N_{k} / \Gamma_{k}$ for all $i \in N_{k}$. UCB then implies that

$$
\xi_{i}\left(v, \Gamma_{\mathcal{P}}\right)-\xi^{k}\left(v_{\{i\}}^{k}, \Gamma_{\mathcal{P}_{\{i\}}^{k}}\right)=\frac{\xi^{k}\left(v, \Gamma_{\mathcal{P}}\right)-\sum_{j \in N_{k}} \xi^{k}\left(v_{\{j\}}^{k}, \Gamma_{\left.\mathcal{P}_{\{j\}}^{k}\right)}\right.}{n_{k}}, \quad \text { for all } i \in N_{k} .
$$

From Step 1 and 2 above, we know $\xi^{k}\left(v, \Gamma_{\mathcal{P}}\right)$ and $\xi^{k}\left(v_{\{j\}}^{k}, \Gamma_{\mathcal{P}_{\{j\}}^{k}}\right)$, for all $j \in N_{k}$. So, equation (4.8) determines $\xi_{i}\left(v, \Gamma_{\mathcal{P}}\right)$ for all $i \in N_{k}$.

Now we proceed by induction similar as in Step 1, but first we show that for each component $C \in N_{k} / \Gamma_{k}$ the total payoff to the players in $C$ is uniquely determined. The payoff $\xi^{k}\left(v, \Gamma_{\mathcal{P}}\right)$ to the a priori union $N_{k}$ has been determined already in Step 1, so

$$
\sum_{i \in N_{k}} \xi_{i}\left(v, \Gamma_{\mathcal{P}}\right)=\xi^{k}\left(v, \Gamma_{\mathcal{P}}\right)
$$

If $N_{k}$ is the unique component in $N_{k} / \Gamma_{k}$, then UCB does not state any requirement. When $N_{k} / \Gamma_{k}$ consists of multiple components, then for every component $C \in N_{k} / \Gamma_{k}$, UCB states that

$$
\frac{\sum_{i \in C} \xi_{i}\left(v, \Gamma_{\mathcal{P}}\right)-\xi^{k}\left(v_{C}^{k}, \Gamma_{\mathcal{P}_{C}^{k}}\right)}{c}=\frac{\xi^{k}\left(v, \Gamma_{\mathcal{P}}\right)-\sum_{K \in N_{k} / \Gamma} \xi^{k}\left(v_{K}^{k}, \Gamma_{\mathcal{P}_{K}^{k}}\right)}{n_{k}} .
$$

\footnotetext{
${ }^{4}$ Note that in the proof of the induction step, every possible spanning tree $\widetilde{\Gamma}$ yields the same solution for the values $\xi^{k}\left(v, \Gamma_{\mathcal{P}}\right), k \in Q$, because otherwise a solution does not exist, which contradicts Theorem 4.1 .
} 
Notice that every payoff $\xi^{k}$ in this equation has been determined in either Step 1 or Step 2. We now prove the induction step similar as in Step 1, and as in [9]. Let $\Gamma_{\mathcal{P}}^{\prime}$ denote the two-level graph structure $\left\langle\Gamma_{M},\left\{\Gamma_{h}^{\prime}\right\}_{h \in M}\right\rangle$ with $\Gamma_{h}^{\prime}=\Gamma_{h}$ if $h \neq k$ and $\Gamma_{k}^{\prime}=\Gamma^{\prime}$ for some graph $\Gamma^{\prime}$ on $N_{k}$. Above we already showed that the payoffs in $N_{k}$ are determined if $\left|\Gamma_{k}\right|=0$. Now, assume that the values $\xi_{i}\left(v, \Gamma_{\mathcal{P}}^{\prime}\right)$ have been determined for every $\Gamma^{\prime}$ with $\left|\Gamma^{\prime}\right|<\left|\Gamma_{k}\right|$. Let $C \in N_{k} / \Gamma_{k}$ be a component in $\left(N_{k}, \Gamma_{k}\right)$. If $C \subseteq N_{k}$ is a singleton set $\{i\}$, then the payoff $\xi_{i}\left(v, \Gamma_{\mathcal{P}}\right)$ of the single player $i \in C$ follows from (4.10). If $c=|C| \geq 2$, then there exists a spanning tree $\left.\widetilde{\Gamma} \subseteq \Gamma_{k}\right|_{C}$ on $C$. So, the number of links in $\widetilde{\Gamma}$ is $c-1$. By UF, for all $\{i, j\} \in \widetilde{\Gamma}$ it holds that

$$
\xi_{i}\left(v, \Gamma_{\mathcal{P}}\right)-\xi_{i}\left(v,\left.\Gamma_{\mathcal{P}}\right|_{-i j} ^{k}\right)=\xi_{j}\left(v, \Gamma_{\mathcal{P}}\right)-\xi_{j}\left(v,\left.\Gamma_{\mathcal{P}}\right|_{-i j} ^{k}\right)
$$

Since $\left|\Gamma_{k} \backslash\{i, j\}\right|=\left|\Gamma_{k}\right|-1$, it follows by the induction hypothesis that all payoffs $\xi_{i}\left(v,\left.\Gamma_{\mathcal{P}}\right|_{-i j} ^{k}\right)$, $\{i, j\} \in \widetilde{\Gamma}$, have been determined. If $C \neq N_{k}$ then the equations (4.10) and (4.11) yield $c$ linearly independent equations in the $c$ unknown payoffs $\xi_{i}\left(v, \Gamma_{\mathcal{P}}\right), i \in C$. If $C=N_{k}$ then the equations (4.9) and (4.11) yield $c$ linearly independent equations in the $c$ unknown payoffs $\xi_{i}\left(v, \Gamma_{\mathcal{P}}\right), i \in C$. Hence, for every $C \in N_{k} / \Gamma_{k}$, all payoffs $\xi_{i}\left(v, \Gamma_{\mathcal{P}}\right), i \in C$, are uniquely determined.

Note that in the proof of Theorem 4.2 we used QCE and QF to determine the sum of the payoffs in every union, similar as done in [9]. In fact, we considered $\Gamma_{M}$ as a one-level graph on $M$. We cannot apply a similar proof using component efficiency to determine the individual payoffs inside each union, because the total payoff to the players in each union should be equal to the total payoff to the union as determined in Step 1, which could be more (or less) than the sum of the payoffs that the components of the communication graph within the union obtain in the internal game. Instead, we applied UCB to obtain uniqueness on the individual level.

We conclude this section by showing that the four axioms are logically independent.

1. [Equal division within the a priori unions] Let the two-level graph game value $\xi^{(1)}$ assign for every $\left\langle v, \Gamma_{\mathcal{P}}\right\rangle \in \mathcal{G}^{N} \times \mathcal{L}_{\mathcal{C}}^{N}$ payoff

$$
\xi_{i}^{(1)}\left(v, \Gamma_{\mathcal{P}}\right)=\frac{\widetilde{v}_{k}\left(N_{k}\right)}{n_{k}},
$$

to every player $i \in N_{k}, k \in M$. This value divides for each a priori union $k \in M$ the worth $\widetilde{v}_{k}\left(N_{k}\right)$ of coalition $N_{k}$ in the restricted quotient game equally amongst the players in $N_{k}$. It satisfies quotient component efficiency, quotient fairness and union fairness but does not satisfy union component balancedness. 
2. [Equal division within the components of the a priori unions] Let the twolevel graph game value $\xi^{(2)}$ assign for every $\left\langle v, \Gamma_{\mathcal{P}}\right\rangle \in \mathcal{G}^{N} \times \mathcal{L}_{\mathcal{C}}^{N}$ payoff

$$
\xi_{i}^{(2)}\left(v, \Gamma_{\mathcal{P}}\right)=\frac{\tilde{\tilde{v}}_{k}(C)}{c}+\frac{\widetilde{v}_{k}\left(N_{k}\right)-\sum_{H \in N_{k} / \Gamma_{k}} \tilde{\tilde{v}}_{k}(H)}{n_{k}},
$$

to every player $i \in C, C \in N_{k} / \Gamma_{k}, k \in M$. Each player $i \in C \in N_{k} / \Gamma_{k}$ gets an equal share in the worth $\tilde{\tilde{v}}_{k}(C)$ of his component and an equal share in the surplus of $N_{k}$ over the sum of the worths of the components in $N_{k} / \Gamma_{k}$. This value satisfies quotient component efficiency, quotient fairness and union component balancedness but it does not satisfy union fairness.

3. [Equal division within the components of the upper-level structure] Let the two-level graph game value $\xi^{(3)}$ be defined for every $\left\langle v, \Gamma_{\mathcal{P}}\right\rangle \in \mathcal{G}^{N} \times \mathcal{L}_{\mathcal{C}}^{N}$ by

$$
\xi_{i}^{(3)}\left(v, \Gamma_{\mathcal{P}}\right)=S h_{i}\left(w_{k(i)}\right), \quad \text { for all } i \in N
$$

where for a priori union $k \in M$ belonging to a component $K \in M / \Gamma_{M}$ of the upperlevel structure, the game $w_{k} \in \mathcal{G}^{N_{k}}$ is defined by

$$
w_{k}(S)= \begin{cases}\tilde{v}_{k}^{\Gamma_{k}}(S), & S \varsubsetneqq N_{k}, \\ \frac{1}{|K|} v(K), & S=N_{k} .\end{cases}
$$

In this case every a priori union $N_{k}$ gets an equal share in the worth of the component to which it belongs in the upper level structure. This value satisfies quotient component efficiency, union fairness and union component balancedness but it does not satisfy quotient fairness.

4. [Efficient total payoff distribution] Let the two-level graph game value $\xi^{(4)}$ be defined for every $\left\langle v, \Gamma_{\mathcal{P}}\right\rangle \in \mathcal{G}^{N} \times \mathcal{L}_{\mathcal{C}}^{N}$ by

$$
\xi_{i}^{(4)}\left(v, \Gamma_{\mathcal{P}}\right)=S h_{i}\left(w_{k(i)}^{*}\right), \quad \text { for all } i \in N
$$

where for a priori union $k \in M w_{k}^{*} \in \mathcal{G}^{N_{k}}$ is defined by

$$
w_{k}^{*}(S)=\left\{\begin{array}{cc}
\tilde{v}_{k}^{\Gamma_{k}}(S), & S \varsubsetneqq N_{k}, \\
S h_{k}(\bar{w}), & S=N_{k},
\end{array}\right.
$$

with game $\bar{w}$ on $M$ defined by $\bar{w}(Q)=v_{\mathcal{P}}^{\Gamma_{M}}(Q)$ for every $Q \varsubsetneqq M$ and $\bar{w}(M)=$ $v_{\mathcal{P}}(M)=v(N)$. In this case the total payoff is equal to the worth $v(N)$ of the grand coalition $N$ of all players, i.e., $\xi^{(4)}$ is efficient. This value $\xi^{(4)}$ satisfies quotient fairness, union fairness and union component balancedness but it does not satisfy quotient component efficiency. 


\section{Comparison with other values}

In this final section we consider several special cases of two-level structure $\Gamma_{\mathcal{P}}$ and its corresponding Owen-type value $\psi$ and show that, for example the Owen value, AumannDrèze value (for games in coalition structure), Myerson value (for communication graph games) and equal surplus division solutions can be obtained as special cases of this value. We distinguish two types of values, one depending on special communication graphs, and the other depending on special partitions.

\subsection{Special communication graphs}

Two special cases of a communication graph are the complete and the empty graph. In this paper these two special cases can occur both on the upper level between the unions as on the lower level within the unions. We first discuss three special cases with an empty graph on the upper level and next three special cases with a complete graph on the upper level.

1. [Empty upper level structure, complete graph within the unions: The Aumann-Drèze value] Consider the case $\Gamma_{\mathcal{P}}$ with $\Gamma_{M}$ the empty graph and every $\Gamma_{k}, k \in M$, the complete graph. In this case every a priori union $N_{k}$ stands alone and the Myerson value applied to the quotient game with empty communication graph assigns to every a priori union $N_{k}, k \in M$, its own payoff $v\left(N_{k}\right)$. In the game $\widetilde{v}_{k}$ on $N_{k}$ every coalition $S \subset N_{k}$ gets its own worth $v(S)$, thus $\widetilde{v}_{k}(S)=v(S)$ for every $S \subseteq N_{k}, k \in M$. Within the union there is no restriction on the cooperation between the players and thus $\tilde{\tilde{v}}_{k}(S)=v(S)$ for every $S \subseteq N_{k}, k \in M$. It follows that

$$
\psi_{i}\left(v, \Gamma_{\mathcal{P}}\right)=S h_{i}\left(\left.v\right|_{N_{k(i)}}\right)=A D_{i}(v, \mathcal{P}), \quad \text { for all } i \in N,
$$

i.e., every player $i$ gets its Shapley value within the subgame of $v$ on the a priori union $N_{k}$ containing $i$, and therefore, in this case the value $\psi$ is equal to the Aumann-Drèze value [1].

2. [Empty two-level structure: Equal surplus division] Consider the case $\Gamma_{\mathcal{P}}$ with both $\Gamma_{M}$ and every $\Gamma_{k}, k \in M$, empty. As in the previous case every a priori coalition $N_{k}, k \in M$, stands alone and gets its own worth $v\left(N_{k}\right)$. Next, within a priori union $N_{k}$ every player $i$ is a stand alone component and $\tilde{\tilde{v}}_{k}(\{i\})=v(\{i\})$ for every $i \in N_{k}$. Then it follows from union component balancedness that for every $k \in M$ and $i \in N_{k}$,

$$
\psi_{i}\left(v, \Gamma_{\mathcal{P}}\right)=v(\{i\})+\frac{v\left(N_{k}\right)-\sum_{i \in N_{k}} v(\{i\})}{n_{k}} .
$$


So, in this case the value $\psi$ assigns within each a priori union $N_{k}$ the equal surplus division solution on the subgame $\left.v\right|_{N_{k}}$, first considered in Driessen and Funaki [5] under the name of the center of the imputation set (CIS-value). In case $v$ is zeronormalized, and thus $v(\{i\})=0$ for every $i \in N$, the value $\psi$ yields the equal division solution within each a priori union $N_{k}$.

3. [Empty upper level structure, connected graphs within the unions: The Myerson value] Consider the case $\Gamma_{\mathcal{P}}$ with $\Gamma_{M}$ the empty graph and every $\Gamma_{k}$, $k \in M$, connected, i.e., for every $k \in M$, union $N_{k}$ itself is the only element in $N_{k} / \Gamma_{k}$. Again every a priori union $N_{k}, k \in M$, stands alone and gets its own worth $v\left(N_{k}\right)$ and in the game $\widetilde{v}_{k}$ every coalition $S \subset N_{k}$ gets its own worth $v(S)$, thus $\widetilde{v}_{k}(S)=v(S)$ for every $S \subseteq N_{k}, k \in M$. Since $\Gamma_{k}$ is connected it follows that $\widetilde{v}_{k}^{\Gamma_{k}}\left(N_{k}\right)=\widetilde{v}_{k}\left(N_{k}\right)=v\left(N_{k}\right)$ and therefore $\tilde{\tilde{v}}_{k}=v^{\Gamma_{k}}$. So $\psi$ yields to every player $i$ in every a priori union $N_{k}$ the payoff of the Myerson value of the subgame on $N_{k}$ with respect to the communication graph $\Gamma_{k}$ within $N_{k}$. Even more, let $\Gamma=\cup_{k \in M} \Gamma_{k}$ be the communication graph between all players obtained by taking the union of all graphs within the unions. Then, by definition every $N_{k}$ is a component of $\Gamma$, i.e., $N / \Gamma=\left\{N_{1}, \ldots, N_{m}\right\}$. By component efficiency of the Myerson value it follows immediately that for the case of an empty upper level structure and connected graphs within the unions, the value $\psi$ is equal to the Myerson value $\mu$ for the game $v$ on $N$ with respect to the (one-level) induced communication stucture $\Gamma=\cup_{k \in M} \Gamma_{k}$ on $N$.

4. [Complete two-level structure: The Owen value] Consider the case $\Gamma_{\mathcal{P}}$ with both $\Gamma_{M}$ and every $\Gamma_{k}, k \in M$, complete graphs. In this case there is no restriction on the cooperation between a priori unions and within the a priori unions. Hence, for every $Q \subseteq M, Q$ is the only component of the subgraph $\left.\Gamma_{M}\right|_{Q}$ and also for every $k$ and every $C \subseteq N_{k}, C$ is the only component of the subgraph $\left.\Gamma_{k}\right|_{C}$. Therefore $\psi$ reduces to the Owen value on $\mathcal{P}: \psi\left(v, \Gamma_{\mathcal{P}}\right)=O w(v, \mathcal{P})$. Notice that in this case quotient component efficiency reduces to efficiency and union component balancedness becomes redundant.

5. [Complete upper level structure, empty graphs within the unions: Equal union surplus division] Consider the case $\Gamma_{\mathcal{P}}$ with $\Gamma_{M}$ the complete graph and $\Gamma_{k}$ the empty graph for every $k \in M$. Again there is no restriction on the cooperation between the unions and therefore

$$
\widetilde{v}_{k}(S)=\bar{v}_{k}(S), \quad \text { for all } k \in M \text { and all } S \subseteq N_{k}
$$

On the other hand, within an a priori union $N_{k}$ every player $i \in N_{k}$ is a stand alone component. With $\tilde{\tilde{v}}_{k}(\{i\})=\bar{v}_{k}(\{i\})$ for all $i \in N_{k}$, and $\tilde{\tilde{v}}_{k}\left(N_{k}\right)=\bar{v}_{k}\left(N_{k}\right)=S h_{k}\left(v_{\mathcal{P}}\right)$ 
the Shapley value of a priori union $k$ in the quotient game, it follows from union component balancedness that, for every $k \in M$ and $i \in N_{k}$

$$
\psi_{i}\left(v, \Gamma_{\mathcal{P}}\right)=\bar{v}_{k}(\{i\})+\frac{S h_{k}\left(v_{\mathcal{P}}\right)-\sum_{i \in N_{k}} \bar{v}_{k}(\{i\})}{n_{k}} .
$$

So, within a priori union $N_{k}$ every player $i$ gets its stand alone value in the game $\bar{v}_{k}$ plus an equal share in the surplus of $N_{k}$ in the quotient game.

6. [Complete upper level structure, connected graphs within the unions: The efficient Myerson-type value of Casajus [4]] Consider the case $\Gamma_{\mathcal{P}}$ with $\Gamma_{M}$ the complete graph and every $\Gamma_{k}, k \in M$, connected. Again $\widetilde{v}_{k}(S)=\bar{v}_{k}(S)$ for all $k \in M$ and $S \subseteq N_{k}$. Because of connectedness of every $\Gamma_{k}$, the value $\psi$ is obtained by applying within every a priori union $N_{k}$ the Myerson value $\mu$ to $\widetilde{v}_{k}=\bar{v}_{k}$ with respect to $\Gamma_{k}$, so for every $k \in M$ and $i \in N_{k}$,

$$
\psi_{i}\left(v, \Gamma_{\mathcal{P}}\right)=\mu_{i}\left(\bar{v}_{k}, \Gamma_{k}\right)
$$

Furthermore, every $\Gamma_{k}$ is connected and by definition $(2.1)$ of $\bar{v}, \bar{v}_{k}^{\Gamma_{k}}\left(N_{k}\right)=\bar{v}_{k}\left(N_{k}\right)=$ $S h_{k}\left(v_{\mathcal{P}}\right)$. Then from the efficiency of the Shapley value it follows that for every $k$, $\sum_{i \in N_{k}} \psi_{i}\left(v, \Gamma_{\mathcal{P}}\right)=S h_{k}\left(v_{\mathcal{P}}\right)$ and $\sum_{i \in N} \psi_{i}\left(v, \Gamma_{\mathcal{P}}\right)=\sum_{k \in M} S h_{k}\left(v_{\mathcal{P}}\right)=v(N)$. So $\psi$ distributes the total worth $v(N)$ and thus meets efficiency.

In fact, in this case the two-level graph game value $\psi$ yields the same payoffs as the socalled CO-value $\phi$, introduced in Theorem 4.2 of Casajus [4] as an efficient alternative for the Myerson value for games with a one-level communication graph. For such a game $\langle v, \Gamma\rangle$ with $\Gamma$ a communication graph on $N$, [4] considers the collection $N / \Gamma$ of components of $\Gamma$ as a cooperation structure $\mathcal{P}$ induced by the communication structure $\Gamma$. Let $N_{k}$ be such a component. Then, within $N_{k}$ the Shapley value is applied to the Myerson restricted game of $\bar{v}_{k}$. This gives the same payoffs as $\psi\left(v, \Gamma_{\mathcal{P}}\right)$ for the two-level structure when $\Gamma_{M}$ is taken to be the complete graph on $M$ and for each $k \in M$ graphs $\Gamma_{k}$ are connected. In this case the Casajus's graph $\Gamma=\cup_{k \in M} \Gamma_{k}$.

\subsection{Special coalition structures}

Finally we discuss the two special cases with respect to the coalition structure.

1. [Partition in singletons: The Myerson value] When $\mathcal{P}=\{\{1\}, \ldots,\{n\}\}$ every a priori union consists of a single player $i$ and there is no game within the unions. 
Hence the value $\psi$ reduces to the Myerson value with respect to the upper level graph structure $\Gamma_{M}$, thus

$$
\psi_{i}\left(v, \Gamma_{\mathcal{P}}\right)=\mu_{i}\left(v, \Gamma_{M}\right)=S h_{i}\left(v^{\Gamma_{M}}\right), \quad \text { for all } i \in N
$$

2. [Single a priori union: The efficient Myerson value] Consider the case $\mathcal{P}=$ $\{N\}$, thus the grand coalition $N$ itself is the singleton a priori union within the coalition structure $\mathcal{P}$. In this case $m=1$ and denoting $\tilde{\tilde{v}}=\tilde{\tilde{v}}_{1}$ and $\Gamma=\Gamma_{1}$ for the single a priori union $N=N_{1}$ in $\mathcal{P}$ we have

$$
\tilde{\tilde{v}}(S)= \begin{cases}v^{\Gamma}(S), & S \varsubsetneqq N, \\ v(N), & S=N .\end{cases}
$$

By definition, the value $\psi$ assigns the Shapley value of the game $\tilde{\tilde{v}}$ on $N$ that equals to the Myerson value of $\langle v, \Gamma\rangle$ plus an equal split of the excess of the worth of the grand coalition over the total worth of all components in graph $\Gamma$, i.e.,

$$
\psi_{i}\left(v, \Gamma_{\mathcal{P}}\right)=\mu_{i}(v, \Gamma)+\frac{v(N)-\sum_{C \in N / \Gamma} v(C)}{n}, \quad \text { for all } i \in N \text {. }
$$

It appears that this is the unique value that satisfies union fairness (within the grand coalition $N$ ) and efficiency. Considering this case as just a one-level communication graph game $\langle v, \Gamma\rangle$ on $N$, recall that the Myerson value is the unique value that satisfies component efficiency and fairness. In fact, in case of $\mathcal{P}=\{N\}$ the value $\psi$ yields the same payoffs as the efficient Myerson-type value of the game $\langle v, \Gamma\rangle$ for games with one-level communication graphs, recently studied in van den Brink, Khmelnitskaya, and van der Laan [3].

\section{References}

[1] Aumann, R.J., J. Drèze (1974), Cooperative games with coalitional structures, International Journal of Game Theory, 3, 217-237.

[2] Brink, R. van den, and G. van der Laan (2005), A class of consistent share functions for cooperative games in coalition structure, Games and Economic Behavior, 51, 193-212.

[3] Brink, R. van den, A. Khmelnitskaya, and G. van der Laan (2011), An efficient and fair solution for communication graph games, TI-Discussion Paper 11-052/1, VU University and Tinbergen Institute, Amsterdam, The Netherlands. 
[4] Casajus, A. (2007), An efficient value for TU games with a cooperation structure, Working paper, Universität Leipzig, Germany.

[5] Driessen, T.S.H., Y. Funaki (1991), Coincidence of and collinearity between game theoretic solutions, OR Spektrum, 13, 15-30.

[6] Kamijo, Y. (2009), A two-step Shapley value for cooperative games with coalition structures, International Game Theory Review, 11, 207-214.

[7] Khmelnitskaya, A. (2010), Graph-restricted games with coalition structures, in: Petrosyan LA, Zenkevich NA (eds.), Contributions to Game Theory and Management, vol. 3, Graduate School of Management SPbU, St. Petersburg, pp. 220-246.

[8] Kongo, T. (2007), Cooperative games with two-level networks, 21COE-GLOPE Working Paper Series, Waseda University, Tokyo. Japan.

[9] Myerson, R.B. (1977), Graphs and cooperation in games, Mathematics of Operations Research, 2, 225-229.

[10] Owen, G. (1977), Values of games with a priori unions, in: Henn R, Moeschlin O (eds.) Essays in mathematical economics and game theory, Springer-Verlag, Berlin, pp. $76-88$.

[11] Shapley, L.S. (1953), A value for $n$-person games, in: Tucker AW, Kuhn HW (eds.) Contributions to the theory of games II, Princeton University Press, Princeton, NJ, pp. 307-317.

[12] Vázquez-Brage, M., I. García-Jurado, and F. Carreras (1996), The Owen value applied to games with graph-restricted communication, Games and Economic Behavior, 12, $42-53$.

[13] Winter, E. (1989) A value for games with level structures, International Journal of Game Theory, 18, 227-242. 